\title{
Exchange-correlation energy densities for two-dimensional systems from quantum dot ground-states
}

\author{
Andreas Wensauer ${ }^{1}$ and Ulrich Rössler ${ }^{1}$ \\ ${ }^{1}$ Institut für Theoretische Physik, Universität Regensburg, D-93040 Regensburg, Germany
}

(Dated: November 21, 2018)

\begin{abstract}
In this paper we present a new approach how to extract polarization-dependent exchangecorrelation energy densities for two-dimensional systems from reference densities and energies of quantum dots provided by exact diagonalization. Compared with results from literature we find systematic corrections for all polarizations in the regime of high densities.
\end{abstract}

PACS numbers: 73.21.-b, 71.10.Ca, 71.15.Mb 


\section{INTRODUCTION}

Density functional theory $(\mathrm{DFT})^{1.2}$ and spin-density functional theory (SDFT) $)^{3.4}$ are powerful techniques to investigate interacting electron systems. However, the results of these methods sensitively depend on the quality of the approximation of the exchangecorrelation (XC) functional by the local (spin) density approximation (L(S)DA) and/or gradient corrections. In contrast to three-dimensional (3D) systems where a large number of parameterizations for XC energy densities and gradient corrections is available the situation is different for two-dimensional (2D) systems. The majority of all calculations for 2D (e.g. for QDs see Refs. $5,6,6,8,9,10,11,12,13]$ ) relies on the parameterization of the XC energy density from Tanatar and Ceperley $\underline{\underline{14}}$ (TC). They numerically calculated XC energy densities as a function of the carrier density (density parameter $r_{s}$ ) and spin polarization $\xi$ by applying variational Monte Carlo and fixed-node Green's function Monte Carlo methods. The results for $\xi=0$ (no polarization) and $\xi=1$ (full polarization) and $r_{s}$-values 1, 5, 10, 15, 20 etc. were fitted and interpolated using a Padé approximation

$$
\varepsilon_{\mathrm{c}}^{\mathrm{TC}}\left(r_{s}, \xi\right) / \mathrm{Ry}=a_{0} \frac{1+a_{1} x}{1+a_{1} x+a_{2} x^{2}+a_{3} x^{3}} \quad\left(x=r_{s}^{1 / 2}\right) .
$$

with parameters $a_{j}$ for $\xi=0$ and 1 respectively (see Tab. IV in Ref. [14]). In the limit of $r_{s} \rightarrow$

0 the asymptotic behavior is characterized by $a_{0}\left[1+b r_{s}+O\left(r_{s}^{3 / 2}\right)\right]$ whereby logarithmic contributions (see ring-diagram summation in Ref. 25]) such as $r_{s} \ln r_{s}$ are not taken into account. The construction of the dependence on the polarization $\xi$ is analogous to the 3D case $^{3.4}$ where the interpolation of the exchange $(\mathrm{X})$ energy in Hartree-Fock approximation $f(\xi)=\left[(1+\xi)^{3 / 2}+(1-\xi)^{3 / 2}-2^{3 / 2}\right] /\left(2-2^{3 / 2}\right)$ is used

$$
\begin{aligned}
\varepsilon_{\mathrm{XC}}^{\mathrm{TC}}\left(r_{s}, \xi\right) / \mathrm{Ry}= & -\frac{4 \sqrt{2}}{3 \pi r_{s}}\left[(1+\xi)^{3 / 2}+(1-\xi)^{3 / 2}\right] \\
& +\varepsilon_{\mathrm{c}}^{\mathrm{TC}}\left(r_{s}, \xi=1\right) / \mathrm{Ry}+\left[\varepsilon_{\mathrm{c}}^{\mathrm{TC}}\left(r_{s}, \xi=0\right)-\varepsilon_{\mathrm{c}}^{\mathrm{TC}}\left(r_{s}, \xi=1\right)\right] f(\xi) / \mathrm{Ry} .
\end{aligned}
$$

Attaccalite, Moroni, Gori-Giorgi, and Bachelet ${ }^{15}$ (AMGB) pursue a similar approach. They calculate the ground state (GS) energy of a 2DEG as a function of $r_{s}$ and $\xi$ with fixednode diffusion Monte Carlo methods which also take into account back-flow correlations. Their parameterization of the XC energy density

$$
\varepsilon_{\mathrm{XC}}^{\mathrm{AMGB}}\left(r_{s}, \xi\right) / \mathrm{Ry}=\varepsilon_{\mathrm{X}}\left(r_{s}, \xi\right) / \mathrm{Ry}+\varepsilon_{\mathrm{C}}\left(r_{s}, \xi\right) / \mathrm{Ry}
$$


comprises the well-known $\mathrm{X}$ energy from the ring-diagram method ${ }^{25}$

$$
\varepsilon_{\mathrm{X}}\left(r_{s}, \xi\right) / \mathrm{Ry}=-\frac{4 \sqrt{2}}{3 \pi r_{s}}\left[(1+\xi)^{3 / 2}+(1-\xi)^{3 / 2}\right]
$$

and the correlation energy

$$
\varepsilon_{\mathrm{C}}\left(r_{s}, \xi\right) / \mathrm{Ry}=\left(\mathrm{e}^{-\beta r_{s}}-1\right) \varepsilon_{\mathrm{X}}^{(6)}\left(r_{s}, \xi\right) / \mathrm{Ry}+2 \alpha_{0}\left(r_{s}\right)+2 \alpha_{1}\left(r_{s}\right) \xi^{2}+2 \alpha_{2}\left(r_{s}\right) \xi^{4}
$$

with

$$
\varepsilon_{\mathrm{X}}^{(6)}\left(r_{s}, \xi\right)=\varepsilon_{\mathrm{X}}\left(r_{s}, \xi\right)-\left(1+\frac{3}{8} \xi^{2}+\frac{3}{128} \xi^{4}\right) \varepsilon_{\mathrm{X}}\left(r_{s}, 0\right)
$$

being the Taylor expansion of the X energy with respect to $\xi$ of the order six and higher. This kind of representation of the XC energy allows for the identification of the term $2 \alpha_{0}\left(r_{s}\right)$ with the correlation energy $\varepsilon_{\mathrm{C}}\left(r_{s}, 0\right)$ and of the term $2 \alpha_{1}\left(r_{s}\right)$ with the spin stiffness. The $\alpha_{i}\left(r_{s}\right)$-parameterizations are generalizations of the Perdew-Wang form ${ }^{26}$ to $2 \mathrm{D}$

$$
\alpha_{i}\left(r_{s}\right)=A_{i}+\left(B_{i} r_{s}+C_{i} r_{s}^{2}+D_{i} r_{s}^{3}\right) \log \left(1+\frac{1}{E_{i} r_{s}+F_{i} r_{s}^{3 / 2}+G_{i} r_{s}^{2}+H_{i} r_{s}^{3}}\right) .
$$

The parameters which were fitted to the numerical results are summarized in Tab. II of Ref. [15].

Another recent work on 2D XC energy densities ${ }^{27}$ is based on a purely analytical approach. The interaction-strength-interpolation (ISI) connects the two limits of strongly and weakly interacting 2DEGs. The result for the XC energy density reads

$$
\begin{aligned}
\varepsilon_{\mathrm{XC}}\left(r_{s}, \xi\right) / \mathrm{Ry}= & 2 \frac{a_{\infty}}{r_{s}}+4 \frac{X\left(r_{s}, \xi\right)}{Y\left(r_{s}, \xi\right)} \times \\
& \times\left[\left(1+Y\left(r_{s}, \xi\right)\right)^{1 / 2}-1-Z(\xi) \log \frac{\left(1+Y\left(r_{s}, \xi\right)\right)^{1 / 2}+Z(\xi)}{1+Z(\xi)}\right] .
\end{aligned}
$$

The functions and parameters entering the interpolation are

$$
\begin{aligned}
& X\left(r_{s}, \xi\right)=\frac{-b_{0}(\xi)}{r_{s}\left[c_{x}(\xi)-a_{\infty}\right]^{2}}, \\
& Y\left(r_{s}, \xi\right)=\frac{4 b_{0}(\xi)^{2} r_{s}}{\left[c_{x}(\xi)-a_{\infty}\right]^{4}}, \\
& Z(\xi)=\frac{-b_{0}(\xi)}{\left[c_{x}(\xi)-a_{\infty}\right]^{3}}-1
\end{aligned}
$$

and

$$
\begin{aligned}
& a_{\infty}=-(2-8 /(3 \pi)) \\
& b_{0}(\xi)=0.1125 \xi^{8}-0.1495 \xi^{6}+0.083 \xi^{4}+0.107 \xi^{2}-0.192 \\
& c_{x}(\xi)=-\frac{2 \sqrt{2}}{3 \pi}\left[(1+\xi)^{3 / 2}+(1-\xi)^{3 / 2}\right] .
\end{aligned}
$$


Two observations can be made with respect to these different forms of the $\mathrm{XC}$ energy densities:

1. To visualize the different parameterizations we show in Fig. 1 the relative deviations, i.e. $\varepsilon_{\mathrm{XC}}^{(1)} / \varepsilon_{\mathrm{XC}}^{(2)}-1$. Figs. 1 (a) and (b) compare the AMGB- and TC-XC energy density on different scales for $r_{s}$. We find a good agreement $\left(\varepsilon_{\mathrm{XC}}^{\mathrm{AMGB}} / \varepsilon_{\mathrm{XC}}^{\mathrm{TC}}-1 \leq 0.008\right)$ which is not surprising as both expressions are based on the same method. On the other hand it is nevertheless remarkable because AMGB explicitly calculated polarizations $0<\xi<1$ whereas TC interpolated over this regime. Larger deviations can be seen for $\varepsilon_{\mathrm{XC}}^{\mathrm{ISI}} / \varepsilon_{\mathrm{XC}}^{\mathrm{TC}}-1$ in (c) and for $\varepsilon_{\mathrm{XC}}^{\mathrm{ISI}} / \varepsilon_{\mathrm{XC}}^{\mathrm{AMGB}}-1$ in (d). There, the relative differences go up to 0.04 , especially for full polarization. This deviation should have its origin in the different applied methods (ISI analytical, TC and AMGB numerical).

2. Besides looking at the difference between the various XC energy densities we can also examine their performance when they are used as input in DFT. In a recent paper ${ }^{28}$ the agreement between SDFT/LSDA (using the TC parameterization) and ED densities for QDs was investigated and found to be fairly good. However this has not to be true for all systems. For example in Fig. 2 we compare GS densities of a QD with $N=8$, $\omega=3 \mathrm{meV}, S=S_{z}=4, L=0(\hbar=1$ in this paper $)$. Especially for the density maximum and minimum the difference between ED and SDFT/LSDA is considerably large. Further, this effect does not depend on the applied parameterization.

These two findings are our motivation to check the accuracy of XC energy densities in 2D by following a new approach. Our starting point are GS densities and energies from ED from which the XC energy densities are extracted in two steps: First we design an iterative scheme to calculate the self-consistent $\mathrm{XC}$ potentials of the KS equations which reproduce the exact densities. The second step deals with the extraction of XC energy densities from these XC potentials assuming $\mathrm{L}(\mathrm{S}) \mathrm{DA}$.

Our paper is organized as follows: We briefly introduce the QD Hamiltonian in Sec. II and the SDFT/LSDA in Sec. III. Then we describe how to extract XC potentials from GS densities (Sec. IV) and XC energy densities from XC potentials (Sec. V). Sec. VI contains the results for systems with arbitrary polarization. We end with a short summary of the most important conclusions in Sec. VII. 


\section{QUANTUM DOT HAMILTONIAN AND GROUND STATE DENSITIES}

We consider a two-dimensional QD with an axially symmetric parabolic confinement potential of strength $\omega_{0}$. As we are especially interested in GS configurations with vanishing angular momentum $L=0$ a magnetic field can be omitted. The Hamiltonian for $N$ particles in real-space representation $\left[\right.$ with $\mathbf{r}=(x, y), \mathbf{p}=\left(p_{x}, p_{y}\right)$ ] reads:

$$
\hat{H}=\sum_{j=1}^{N}\left(\frac{\mathbf{p}_{j}^{2}}{2 m^{*}}+\frac{1}{2} m^{*} \omega_{0}^{2} \mathbf{r}_{j}^{2}\right)+\frac{1}{2} \sum_{j, k=1}^{N}{ }^{\prime} \frac{e^{2}}{4 \pi \varepsilon \varepsilon_{0}\left|\mathbf{r}_{j}-\mathbf{r}_{k}\right|}
$$

Here $m^{*}$ is the effective mass, $e$ is the electron charge, and $\varepsilon$ is the screening constant of the host semiconductor.

In order to get the reference densities and energies for the investigation of $\mathrm{XC}$ energy densities we apply ED techniques which provide results of high accuracy. ${ }^{29}$ Please note that the (spin-)density of all eigenstates of the angular momentum operator are functions of radius $r$ but not of the angle $\varphi$. Therefore, the relevant quantities from ED are the spin-densities $n_{\sigma}(r)$ of the GS and its energy $E_{0}$.

\section{SPIN-DENSITY FUNCTIONAL THEORY AND LOCAL (SPIN-)DENSITY APPROXIMATION}

In this Section we sketch the basics of $(\mathrm{S}) \mathrm{DFT}$ and L(S)DA. The DFT formalism was originally established by Hohenberg, Kohn, and Sham ${ }^{1,2}$ and generalized to spin-polarized systems ${ }^{3}$ by including the coupling of the polarization to an applied magnetic field. Accordingly, the Hohenberg-Kohn (HK) theorem has to be modified with respect to the spin degrees of freedom ${ }^{3}$. For this case, it states that two different non-degenerate ground-state wavefunctions $|\Psi\rangle$ and $\left|\Psi^{\prime}\right\rangle$ always yield different combinations $\left(n_{\sigma}(\mathbf{r})\right) \neq\left(n_{\sigma}^{\prime}(\mathbf{r})\right)$ of spin densities. This is sufficient to establish a functional of the total energy with the usual functional properties

$$
E_{V_{\sigma}}\left[n_{\sigma}\right]=F_{\mathrm{HK}}\left[n_{\sigma}\right]+\sum_{\sigma} \int \mathrm{d} \mathbf{r} V_{\sigma}(\mathbf{r}) n_{\sigma}(\mathbf{r})
$$

and the universal HK functional

$$
F_{\mathrm{HK}}\left[n_{\sigma}\right]=\left\langle\Psi\left[n_{\sigma}\right]|T+W| \Psi\left[n_{\sigma}\right]\right\rangle
$$

Thus, even in the limit of vanishing magnetic fields the SDFT scheme can yield a spinpolarized ground state for even electron numbers due to Hund's rule..$^{6.30}$ 
For practical purposes the variational scheme has to be mapped on the Kohn-Sham (KS) system, i.e. an effective single-particle system with the same GS densities as the interacting system. The spin-degree of freedom is considered in the KS equations ${ }^{3}$ by assuming the total spin $S_{z}$ in $z$-direction to be a good quantum number

$$
\left\{-\frac{\hbar^{2}}{2 m^{*}} \nabla^{2}+V_{\sigma}(\mathbf{r})+\frac{e^{2}}{4 \pi \varepsilon \varepsilon_{0}} \int \mathrm{d} \mathbf{r}^{\prime} \frac{n\left(\mathbf{r}^{\prime}\right)}{\left|\mathbf{r}-\mathbf{r}^{\prime}\right|}+V_{\mathrm{XC}, \sigma}\left(\left[n_{\sigma}\right], \mathbf{r}\right)\right\} \varphi_{j, \sigma}(\mathbf{r})=\varepsilon_{j, \sigma} \varphi_{j, \sigma}(\mathbf{r})
$$

with the spin $\sigma= \pm$ in $z$-direction and the $\mathrm{KS}$ energies $\varepsilon_{1, \sigma} \leq \varepsilon_{2, \sigma} \leq \ldots$. For a system containing $N$ particles the spin densities are given by

$$
n_{\sigma}(\mathbf{r})=\sum_{j} \gamma_{j, \sigma}\left|\varphi_{j, \sigma}(\mathbf{r})\right|^{2}
$$

with $\gamma_{j, \sigma}$ being occupation numbers of the KS levels in the ground state $\left(\sum_{j} \gamma_{j, \sigma}=N_{\sigma}\right.$ and $\left.N_{+}+N_{-}=N\right)$. Then the GS density and polarization are

$$
\begin{aligned}
& n(\mathbf{r})=n_{+}(\mathbf{r})+n_{-}(\mathbf{r}) \\
& \xi(\mathbf{r})=\frac{n_{+}(\mathbf{r})-n_{-}(\mathbf{r})}{n(\mathbf{r})}
\end{aligned}
$$

The XC potentials

$$
V_{\mathrm{XC}, \sigma}\left(\left[n_{\sigma}\right], \mathbf{r}\right)=\frac{\delta E_{\mathrm{XC}}\left[n_{\sigma}\right]}{\delta n_{\sigma}(\mathbf{r})}
$$

are defined as functional derivatives of the XC energy functional

$$
E_{\mathrm{XC}}\left[n_{\sigma}\right]=F_{\mathrm{HK}}\left[n_{\sigma}\right]-\frac{1}{2} \frac{e^{2}}{4 \pi \varepsilon \varepsilon_{0}} \int \mathrm{d} \mathbf{r} \int \mathrm{d} \mathbf{r}^{\prime} \frac{n(\mathbf{r}) n\left(\mathbf{r}^{\prime}\right)}{\left|\mathbf{r}-\mathbf{r}^{\prime}\right|}-T_{\mathrm{S}}\left[n_{\sigma}\right] .
$$

$\left(T_{\mathrm{S}}\left[n_{\sigma}\right]\right.$ denotes the kinetic energy functional of the KS system.) The total ground-state energy $E_{0}$ of the interacting system can be calculated from

$$
E_{0}=\sum_{j, \sigma} \gamma_{j, \sigma} \varepsilon_{j, \sigma}-\frac{1}{2} \frac{e^{2}}{4 \pi \varepsilon \varepsilon_{0}} \int \mathrm{d} \mathbf{r} \int \mathrm{d} \mathbf{r}^{\prime} \frac{n(\mathbf{r}) n\left(\mathbf{r}^{\prime}\right)}{\left|\mathbf{r}-\mathbf{r}^{\prime}\right|}-\sum_{\sigma} \int \mathrm{d} \mathbf{r} V_{\mathrm{XC}, \sigma}\left(\left[n_{\sigma}\right], \mathbf{r}\right) n_{\sigma}(\mathbf{r})+E_{\mathrm{XC}}\left[n_{\sigma}\right]
$$

Concerning the $\mathrm{XC}$ potentials we apply the $\mathrm{L}(\mathrm{S}) \mathrm{DA}$

$$
E_{\mathrm{XC}}\left[n_{\sigma}\right] \approx \int \mathrm{d} \mathbf{r} n(\mathbf{r}) \varepsilon_{\mathrm{XC}}\left(n_{+}(\mathbf{r}), n_{-}(\mathbf{r})\right)
$$

The most important parameterizations for the XC energy density $\varepsilon_{\mathrm{XC}}\left(n_{+}, n_{-}\right)\left(\right.$or $\left.\varepsilon_{\mathrm{XC}}\left(r_{s}, \xi\right)\right)$ used in 2D calculations were introduced in Sec. I. 


\section{CALCULATION OF XC POTENTIALS}

The first step of our concept to obtain XC energy densities for $2 \mathrm{D}$ systems is the calculation of XC potentials from GS densities. Before presenting our method we will briefly review the literature on the inversion of the $\mathrm{KS}$ equations for $3 \mathrm{D}$ systems. In a pioneer work Almbladh and Pedroza adapt parametrized XC potentials to the electron densities of light atoms. ${ }^{16}$ An alternative approach by Aryasetiawan and Stott formulates the problem in terms of $(N-1)$ coupled non-linear differential equations. ${ }^{17}$ Holas and March derive a solution by applying the Pauli potential and energy and $(N-1)$ Euler equations leading to a differential equation (DEQ) for the density amplitude $\sqrt{n(\mathbf{r})} \cdot \frac{18}{6}$ Görling describes an approach based on the linear response of potentials on small density modifications. ${ }^{19}$ An iterative method to construct $\mathrm{KS}$ orbitals and $\mathrm{XC}$ potentials for a given electron density is presented by Wang and Parr ${ }^{20}$. They use the inverted KS-Schrödinger equation to generate an improved effective potential for the next iteration. In another paper which mainly focuses on kinetic energy functionals Zhao et al. propose a method which is based on Lagrange multipliers to gain XC potentials. ${ }^{21}$ Tozer et al. train neural networks ${ }^{23}$ using XC potentials calculated with Zhao et al.'s method and determine fit parameters for XC functionals ${ }^{24}$. Following Ref. 20] Leeuwen and Baerends present a modified form of the inverted KSSchrödinger equation ${ }^{22}$ which will also be applied for a $2 \mathrm{D}$ system in the present paper and be discussed in detail below.

The KS-Hamiltonian of axially symmetric 2D QDs reads

$$
H_{\mathrm{S}, \sigma}=-\frac{\hbar^{2}}{2 m^{*}}\left[\frac{1}{r} \frac{\partial}{\partial r}\left(r \frac{\partial}{\partial r}\right)+\frac{1}{r^{2}} \frac{\partial^{2}}{\partial \varphi^{2}}\right]+\frac{1}{2} m^{*} \omega_{0}^{2} r^{2}+V_{\mathrm{H}}(r)+V_{\mathrm{XC}, \sigma}\left(\left[n_{\sigma}\right], r\right)
$$

Inversion of the $\mathrm{KS}$ equations means the calculation of the exact $\mathrm{XC}$ potentials up to a gauge-constant $c_{\sigma}$

$$
V_{\mathrm{XC}, \sigma}(r):=V_{\mathrm{XC}, \sigma}\left(\left[n_{\sigma}\right], r\right)+c_{\sigma}
$$

of a system with given electron spin densities $n_{\sigma}(r)$, i.e. we want to find the self-consistent solution of the KS-Schrödinger equation (26) under the constraint $n_{\sigma}(r)=n_{\mathrm{KS}, \sigma}(r)$. The iteration scheme $\frac{22}{2}$ is based on a method presented in Ref. 20]. It should not be considered as a strict proof but as plausibility argument.

In a first step we split off the scalar XC potential from the KS-Hamiltonian (26) and 
denote the rest by $\hat{H}_{0, \sigma}$

$$
\hat{H}_{\mathrm{S}, \sigma}=\hat{H}_{0, \sigma}+\hat{V}_{\mathrm{XC}, \sigma} .
$$

Using the eigenfunctions $\varphi_{j, \sigma}(\mathbf{r})$ and the eigenvalues $\varepsilon_{j, \sigma}$ of the KS-Hamiltonian we obtain the link between exact spin densities and exact XC-potentials

$$
V_{\mathrm{XC}, \sigma}(r)=\frac{1}{n_{\sigma}(r)} \sum_{j} \gamma_{j, \sigma} \varphi_{j, \sigma}^{*}(\mathbf{r})\left(\varepsilon_{j, \sigma}-H_{0, \sigma}\right) \varphi_{j, \sigma}(\mathbf{r}) .
$$

We denote eigenfunctions, eigenvalues, and densities of the $k$-th iteration step by $\varphi_{j, \sigma}^{(k)}(\mathbf{r}), \varepsilon_{j, \sigma}^{(k)}$, and $n_{\mathrm{KS}, \sigma}^{(k)}(r)$, i.e. they are solutions of the KS-Schrödinger equation with the KS-Hamiltonian $\hat{H}_{\mathrm{S}, \sigma}^{(k-1)}=\hat{H}_{0, \sigma}+\hat{V}_{\mathrm{XC}, \sigma}^{(k-1)}$. Thus, we are able to construct the XC potential for the next step by applying Eq. (29)

$$
V_{\mathrm{XC}, \sigma}^{(k)}(r)=\frac{1}{n_{\sigma}(r)} \sum_{j} \gamma_{j, \sigma} \varphi_{j, \sigma}^{(k)^{*}}(\mathbf{r})\left(\varepsilon_{j, \sigma}^{(k)}-H_{0, \sigma}\right) \varphi_{j, \sigma}(\mathbf{r})^{(k)} .
$$

With wavefunctions $\varphi_{j, \sigma}^{(k)}(\mathbf{r})$ being solutions of $\hat{H}_{\mathrm{S}, \sigma}^{(k-1)}$, the iteration scheme for scalar potentials 22 is given by

$$
V_{\mathrm{XC}, \sigma}^{(k)}(r)=V_{\mathrm{XC}, \sigma}^{(k-1)}(r) \frac{n_{\mathrm{KS}, \sigma}^{(k-1)}(r)}{n_{\sigma}(r)} .
$$

During the iteration we will assume $V_{\mathrm{XC}, \sigma}^{(k)}>0$. If the KS density in the $(k-1)$-th iteration step is locally too high (low) the potential of step $k$ will be reduced (increased) at the same place. Consequently, the new density will be larger (smaller). However, the gauge constants $c_{\sigma}$ of the potentials $V_{\mathrm{XC}, \sigma}^{(k)}$ cannot be determined by this method (see Ref. 31]). We will discuss this problem in detail in the following Section.

In the context of the iteration process the aspect of representability of the exact GS density is also tested: As result of a converging iteration we obtain an effective potential so that the KS density reproduces the density of the exact system.

We tested the numerical procedure for a QD with six electrons and a confinement potential of $3 \mathrm{meV}$. In the GS the two lowest shells are occupied, i.e. the system is unpolarized and the paramagnetic current density vanishes due to $L=0$. In this test calculation we started from a GS density which was calculated using conventional DFT/LDA and TC parameterization for XC energies. Thus, we could exclude any problems arising from representability. The result of this test calculation shows perfect agreement between initial and final densities and effective potentials proving the validity of our program. 


\section{CALCULATION OF XC ENERGY DENSITIES}

In this Section we focus on the second step of the system, the extraction of $\mathrm{XC}$ energy densities from exact XC potentials. In order to establish a relation between these two quantities we apply the L(S)DA (25) on the general Eq. (23) for the functionals and obtain

$$
V_{\mathrm{XC}, \sigma}(r)=\varepsilon_{\mathrm{XC}}(r)+n(r) \frac{\partial \varepsilon_{\mathrm{XC}}}{\partial n_{\sigma}}(r) .
$$

We solve for $\frac{\partial \varepsilon_{\mathrm{XC}}}{\partial n_{\sigma}}(r)$

$$
\frac{\partial \varepsilon_{\mathrm{XC}}}{\partial n_{\sigma}}(r)=\frac{1}{n(r)}\left(-\varepsilon_{\mathrm{XC}}(r)+V_{\mathrm{XC}, \sigma}(r)\right)
$$

and plug the result into the derivative of the $\mathrm{XC}$ energy density as a function of the radius

$$
\frac{\partial \varepsilon_{\mathrm{XC}}(r)}{\partial r}=\sum_{\sigma} \frac{\partial \varepsilon_{\mathrm{XC}}}{\partial n_{\sigma}} \frac{\partial n_{\sigma}}{\partial r}
$$

After some algebra we arrive at a linear DEQ

$$
\frac{\partial \varepsilon_{\mathrm{XC}}(r)}{\partial r}+\frac{\partial \log n}{\partial r}(r) \varepsilon_{\mathrm{XC}}(r)=I(r)
$$

with the inhomogeneity

$$
I(r)=\frac{1}{n(r)} \sum_{\sigma}\left(V_{\mathrm{XC}, \sigma}(r)+c_{\sigma}\right) \frac{\partial n_{\sigma}}{\partial r}(r)
$$

which contains all the information about the dependence on the spin densities. In Eq. (36) we take into account that the gauge constants $c_{\sigma}$ of the scalar potentials are not known from the previous step. They will be calculated later. The solution of the homogeneous part of DEQ (35) is given by $\varepsilon_{\mathrm{XC}}^{\text {hom }}(r)=\alpha / n(r)$, a special solution can be calculated using the ansatz $\varepsilon_{\mathrm{XC}}^{\mathrm{spez}}(r)=\beta(r) / n(r)$. The function $\beta(r)$ results from an elementary DEQ

$$
\frac{\partial \beta}{\partial r}(r)=n(r) I(r),
$$

whose solution is

$$
\beta(r)=\int_{0}^{r} \mathrm{~d} r^{\prime} n\left(r^{\prime}\right) I\left(r^{\prime}\right)-\beta(0) .
$$

Thus the general solution of DEQ (35) is

$$
\varepsilon_{\mathrm{XC}}(r)=\varepsilon_{\mathrm{XC}}^{\mathrm{hom}}(r)+\varepsilon_{\mathrm{XC}}^{\mathrm{spez}}(r)=\frac{\alpha-\beta(0)}{n(r)}+\frac{1}{n(r)} \int_{0}^{r} \mathrm{~d} r^{\prime} n\left(r^{\prime}\right) I\left(r^{\prime}\right) .
$$


After substituting the inhomogeneity the result reads

$$
\varepsilon_{\mathrm{XC}}(r)=\frac{\alpha-\beta(0)-n_{\sigma}(0) \sum_{\sigma} c_{\sigma}}{n(r)}+\sum_{\sigma} c_{\sigma} \frac{n_{\sigma}(r)}{n(r)}+\frac{1}{n(r)} \int_{0}^{r} \mathrm{~d} r^{\prime} \sum_{\sigma} V_{\mathrm{XC}, \sigma}\left(r^{\prime}\right) \frac{\partial n_{\sigma}}{\partial r^{\prime}}\left(r^{\prime}\right) .
$$

With the modulus of the XC energy being finite in $\mathrm{L}(\mathrm{S}) \mathrm{DA}$

$$
\left|E_{\mathrm{XC}}\left[n_{\sigma}\right]\right| \approx\left|\int \mathrm{d} \mathbf{r} n(r) \varepsilon_{\mathrm{XC}}(r)\right|=\int \mathrm{d} \mathbf{r} n(r)\left|\varepsilon_{\mathrm{XC}}(r)\right|<\infty
$$

we choose $\alpha-\beta(0)-n_{\sigma}(0) \sum_{\sigma} c_{\sigma}=0$ thus avoiding any divergent contributions. Consequently the analytical solution of (35) satisfying the physical boundary conditions is

$$
\varepsilon_{\mathrm{XC}}(r)=\sum_{\sigma} c_{\sigma} \frac{n_{\sigma}(r)}{n(r)}+\frac{1}{n(r)} \int_{0}^{r} \mathrm{~d} r^{\prime} \sum_{\sigma} V_{\mathrm{XC}, \sigma}\left(r^{\prime}\right) \frac{\partial n_{\sigma}}{\partial r^{\prime}}\left(r^{\prime}\right) .
$$

The last step is the calculation of the gauge constants $c_{\sigma}$ of the scalar potentials. One condition which has not been used yet is the agreement of the DFT GS energy and the exact GS energy. If we eliminate $V_{\mathrm{XC}, \sigma}(\mathbf{r})$ in Eq. (24) and write it in a modified form

$$
E_{G Z}=\sum_{j \sigma} \gamma_{j \sigma} \int \mathrm{d} \mathbf{r} \varphi_{j \sigma}^{*}(\mathbf{r})\left(\frac{\mathbf{p}^{2}}{2 m^{*}}+V_{\sigma}(\mathbf{r})\right) \varphi_{j \sigma}(\mathbf{r})+\frac{1}{2} \int \mathrm{d} \mathbf{r} V_{\mathrm{H}}(\mathbf{r}) n(\mathbf{r})+E_{\mathrm{XC}}\left[n_{\sigma}\right]
$$

we can calculate the (exact!) $\mathrm{XC}$ energy $E_{\mathrm{XC}}\left[n_{\sigma}\right]$ for the GS densities $\left(n_{\sigma}\right)$ : the KSwavefunctions are known from the selfconsistent solution of the KS equations (see IV). Thus, the expectation values $\int \mathrm{d} \mathbf{r} \varphi_{j \sigma}^{*}(\mathbf{r})\left(\frac{1}{2 m^{*}} \mathbf{p}^{2}+V_{\sigma}(\mathbf{r})\right) \varphi_{j \sigma}(\mathbf{r})$ of the non-interacting system can be calculated. The Coulomb energy and the GS-energy are uniquely determined by the interacting system. On the other hand the XC energy for QDs in $\mathrm{L}(\mathrm{S}) \mathrm{DA}$ is given by

$$
E_{\mathrm{XC}}\left[n_{\sigma}\right] \approx \int \mathrm{d} \mathbf{r} n(r) \varepsilon_{\mathrm{XC}}(r)=\sum_{\sigma} c_{\sigma} N_{\sigma}+\int_{0}^{\infty} \mathrm{d} r \int_{0}^{r} \mathrm{~d} r^{\prime} \sum_{\sigma} V_{\mathrm{XC}, \sigma}\left(r^{\prime}\right) \frac{\partial n_{\sigma}}{\partial r^{\prime}}\left(r^{\prime}\right),
$$

what makes $\sum_{\sigma} c_{\sigma} N_{\sigma}$ accessible. In the case of unpolarized systems $c:=c_{\uparrow}=c_{\downarrow}$ and for full polarization $c_{\sigma}$ of the unoccupied spin direction is irrelevant (as the corresponding $N_{\sigma}=0$ ). Then the results for the XC energy density are unique. For partially polarized systems uniqueness of the results can be achieved by additionally demanding asymptotic agreement of $V_{\mathrm{XC}, \sigma}(r)$ for finite systems 31

$$
\lim _{r \rightarrow \infty} V_{\mathrm{XC}, \uparrow}(r)=\lim _{r \rightarrow \infty} V_{\mathrm{XC}, \downarrow}(r) .
$$

As a result of the inversion of the $\mathrm{L}(\mathrm{S}) \mathrm{DA}$ formalism we obtain the $\mathrm{XC}$ energy density as a function of the radius. After eliminating the radius by using the spin densities $n_{\sigma}(r)$, or alternatively $r_{s}(r)$ and $\xi(r)$, we arrive at the standard representation of the XC energy density as a function of $\left(r_{s}, \xi\right)$. 


\section{RESULTS}

After introducing the methodology in the two previous Sections we summarize the numerical results for $\mathrm{XC}$ potentials and $\mathrm{XC}$ energy densities here. The investigated systems are classified due to their degree of polarization. For unpolarized $(\xi=0)$ and fully polarized systems $(\xi=1)$ the $\mathrm{XC}$ energy density is only a function of the density parameter $r_{s}$ whereas for partially polarized systems $\varepsilon_{\mathrm{XC}}$ depends on both $r_{s}$ and polarization $\xi$. But first we will examine an analytically solvable system for two electrons to study the asymptotics of $\mathrm{XC}$ potentials.

\section{A. The two-electron system}

The system with two electrons will be used to find the asymptotic behavior of densities, KS wavefunctions, and XC potentials as QD helium can be calculated analytically for special values of the confinement potential. ${ }^{37}$ For a system with confinement energy $\omega=2$ Ry the analytical expression for the singlet GS wavefunction (GS energy 6 Ry) reads

$$
\begin{aligned}
\Psi\left(\mathbf{r}_{1}, \mathbf{r}_{2}\right) & =\varphi_{\mathrm{CM}}\left(\left|\mathbf{r}_{1}+\mathbf{r}_{2}\right| / 2\right) \varphi_{\text {rel }}\left(\left|\mathbf{r}_{1}-\mathbf{r}_{2}\right|\right) \\
& =C_{\mathrm{CM}} \mathrm{e}^{-\left|\mathbf{r}_{1}+\mathbf{r}_{2}\right|^{2} / 4} \cdot C_{\mathrm{rel}}(1+r) \mathrm{e}^{-\left|\mathbf{r}_{1}-\mathbf{r}_{2}\right|^{2} / 4}
\end{aligned}
$$

with $C_{\mathrm{CM}}=\sqrt{2 / \pi}$ and $C_{\text {rel }}=1 / \sqrt{2 \pi(\sqrt{2 \pi}+3)} \cdot \frac{37}{}$ Consequently, we are also able to calculate the density, the Hartree potential and the XC potential analytically. As the full expressions of these quantities are very complex and do not give much insight we restrict ourselves to the formulas which yield the correct asymptotics for the limit of large radii. The density decays exponentially with

$$
\lim _{r \rightarrow \infty} n(r)=2 \pi C_{\mathrm{CM}}^{2} C_{\mathrm{rel}}^{2} r^{2} \mathrm{e}^{-r^{2}}
$$

and the Hartree potential for large $r$ is that of a point charge

$$
\lim _{r \rightarrow \infty} V_{\mathrm{H}}(r)=4 / r
$$

Thus, the asymptotics of the KS wavefunction $R(r)=\sqrt{\pi n(r)}$ is

$$
\lim _{r \rightarrow \infty} R(r)=\sqrt{2} \pi C_{\mathrm{CM}} C_{\mathrm{rel}} r \mathrm{e}^{-r^{2} / 2} .
$$


$R(r)=\sqrt{\pi n(r)}$ can be used to calculate the $\mathrm{XC}$ potential

$$
V_{\mathrm{XC}}(r) / \mathrm{Ry}=\varepsilon_{0}-r^{2}-V_{\mathrm{H}}(r)+\frac{\left[\frac{1}{r} \frac{\partial}{\partial r}\left(r \frac{\partial}{\partial r}\right)\right] R(r)}{R(r)} .
$$

( $r^{2}$ is the parabolic confinement in Ry.) The KS energy $\varepsilon_{0}=4$ Ry was chosen so that the the $\mathrm{XC}$ potential vanishes in the limit $r \rightarrow \infty$

$$
\lim _{r \rightarrow \infty} V_{\mathrm{XC}}(r)=-2 / r+1 / r^{2}
$$

The leading term $-2 / r$ is a manifestation of the strong selfinteraction in the case of two

electrons. The selfinteraction potential is exactly compensated by the $\mathrm{X}$ potential $V_{\mathrm{X}}(r)=$ $V_{\mathrm{H}}(r) / 2$ in the case of a singlet configuration. ${ }^{38}$

In summary we found from this analytical calculation that the asymptotic behavior of the $\mathrm{XC}$ potential is a rational function which converges to zero. $\mathrm{XC}$ energy densities cannot be extracted from this kind of system because of the dominating selfinteraction contribution.

\section{B. Unpolarized systems}

Unpolarized configurations without angular momentum are typically closed-shell systems with 2, 6, 12, etc. electrons. Unfortunately, the GS densities from ED for 12 and more particles are not convergent so far, so that they cannot be used as reference densities at present. On the other hand, ED will give excellent results for two particles. However, predominating self-interaction effects in a two-electron system are a problem for the extraction of reliable $\mathrm{XC}$ energy densities. Therefore, we will focus on the six-electron system with two closed shells.

To illustrate the inversion of the KS equations we investigate a six-electron system with a standard confinement potential $3.32 \mathrm{meV}$ (see Fig. 3). The reference GS density from ED is depicted in (a). The quality of the ED results was tested using an increasing number of Slater determinants. For the present calculation with 326120 Slater determinants we found a good convergence of GS densities up to a radius of $8 \mathrm{a}_{0}$.

By applying the iteration scheme (31) we are now able to calculate the XC potential up to a constant (see black dotted line in (b)) which exactly reproduces the reference density from ED. For reasons of comparison there is also the TC XC-energy density plotted in (b) which was calculated with the reference density. In the next step we solve the DEQ (35) to 
calculate the $\mathrm{XC}$ energy density as a function of the radius and determine the gauge constant $c_{\sigma}$. The extracted XC energy density (black dashed line) is shown together with the TC XC energy density (grey solid) in (c). In (d) the same two curves are plotted, however, we eliminated the radius in favor of the density parameter. The redundancy in the extracted $\mathrm{XC}$ energy density (black, dashed curve) is explained as follows: Close to the local density maximum we get two $\varepsilon_{\mathrm{XC}}$ values per density value as reflected in Fig. 3 (d). The deviation between the two values will be discussed later.

First let us focus on the difference in the high-density region in the center and in the low-density regime at the edge (see Fig. [3). While the results for high densities are reliable, the differences at the edge of the dot are a consequence of the finite basis set in ED. However, in the analytically solvable model for two electrons the XC potential (501) converges to zero, whereas this is not true for the ED result (see Fig. [3(b)). Consequently, the problem emerges again in the XC energy density (see Fig. [3(c), (d)). Thus, we can reassure that the behavior of $V_{\mathrm{XC}}(r)$ at the edge is not due to the divergence of $(\partial n(r) / \partial r) / n(r)$ or non-local effects. However, we want to emphasize that the difficulties of XC energy densities at the edge do not affect the accuracy of the gauge constant $c_{\sigma}$ as in Eq. (43) $\varepsilon_{\mathrm{XC}}(r)$ is weighted with the exponentially decreasing density.

Now let us turn to the redundant structure in $\varepsilon_{\mathrm{XC}}$ of Fig. [3.(d). As the XC energy densities were extracted under the assumption of LDA the order of magnitude of non-local effects is mapped in Fig. [3(d). These problems have to be taken into account if we interpret the results of extracted XC energy densities. The best test for the reliability is the calculation

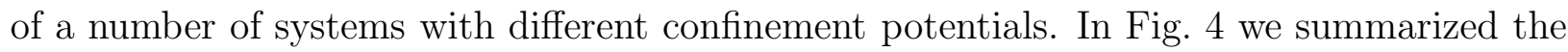
extracted $\varepsilon_{\mathrm{XC}}$ from systems with confinement potentials between $3 \mathrm{meV}$ and $100 \mathrm{meV}$. The $\varepsilon_{\mathrm{XC}}$ were taken from the center and the density maximum. Thus, they are not influenced by any edge effects and in addition they represent upper and lower boundaries for the extracted XC energy densities (see Fig. [3 (d)). All curves show the difference between a specific XC energy density and the TC XC energy density. While the other parameterizations from literature (AMGB, ISI) do not deviate too much from the TC reference both, the upper and lower bound of extracted XC energy densities exhibit systematic corrections in the regime of small density parameters or high densities. This trend is stable and consistent for many different strengths of confinement potentials and therefore it is reliable. The tendency to smaller XC energy densities is also consistent with an increasing difference of DFT and 
ED-GS energies with growing external confinement.

\section{Fully polarized systems}

In the case of full polarization we consider the configurations with $N=6, S=3, L=0$ and $N=8, S=4, L=0$ (see Fig. 2 for $N=8$ ). In analogy to the unpolarized systems we invert the KS equations and solve the DEQ (35). The resulting $\mathrm{XC}$ energy densities from the extrema (center, maximum, minimum) are plotted in Fig. 5] (a) for six electrons and in (b) for eight electrons. The strength of the confinement potential varies between $3 \mathrm{meV}$ and $100 \mathrm{meV}$. The plots show again the deviation from the TC parameterization. As in the case of unpolarized systems we find for six and eight electrons systematic corrections in the high density regime towards smaller XC energy densities. In Fig. 5 (c) we check the consistency of the extracted XC energy densities by comparing not only results for one system with different confinement potentials but also different systems. The good agreement is an important proof for the quality and the reliability of our results.

\section{Partially polarized systems}

Finally we investigate the partially polarized systems $N=4, S=1, L=0$ and $N=6$, $S=2, L=0$. As an example we consider four electrons in a confinement potential of $3 \mathrm{meV}$ (see Fig. 6). The spin-down and spin-up Fig. 6(a) densities from ED are exactly reproduced by the XC potentials plotted as dashed, black lines in Fig. 6 (b). Please note that the XC potentials for the two spin-directions exhibit the same asymptotic behavior as demanded. The grey, solid lines in (b) are the (gauged) TC-XC potentials as they can be calculated from the spin-densities in (a). After solving the DEQ (35) we are able to plot the XC energy density as a function of the radius (see (d)). However, for partial polarization we have to take into account that $\varepsilon_{\mathrm{XC}}$ depends on both, $r_{s}$ and $\xi$.

In order to plot the results (Fig. [7) we have to collect all combinations $\left(r_{s}, \varepsilon_{\mathrm{XC}}\right)$ for given polarization $\xi$ from the systems with four and six electrons and confinement energies from $3 \mathrm{meV}$ to $100 \mathrm{meV}$. All the values were taken from areas with a reasonably high density. Only in this regime results are reliable as follows from the same arguments as for unpolarized and fully polarized systems. The summary of extracted XC energy densities is shown in Fig. 7 
for the polarizations $\xi=0.2(\mathrm{a}), \xi=0.4(\mathrm{~b}), \xi=0.6$ (c), and $\xi=0.8$ (d) as deviation from the TC parameterization. Similar to unpolarized and fully polarized systems we find systematic corrections for small $r_{s}$ compared to TC, AMGB, and ISI. The reliability of the extracted $\varepsilon_{\mathrm{XC}}$ is emphasized by the fact that the difference between them is not larger than for $\xi=0$ and $\xi=1$.

\section{SUMMARY AND OUTLOOK}

In this paper we present a new approach how to extract 2D-XC energy densities from GS densities and energies of QDs calculated by ED. Our focus was on configurations with arbitrary polarization but without paramagnetic current density. In comparison with parameterizations from literature ( $\mathrm{TC}^{14}, \mathrm{AMGB}^{15}$, ISI $\left.{ }^{27}\right)$ we find systematic corrections in the regime of small density parameters or high densities for all polarizations. This result was consistently accomplished by exploiting the redundancy arising from different configurations and/or different strengths of the external confinement potentials.

However, our results are restricted to a small range of density parameters. Therefore, it would be useful to extend the calculations to more confinement potentials or to confirm them by an alternative method which also provides an approach for an improved parameterization.

Another open question is the role of the corrections due to the paramagnetic current density or vorticity ${ }^{32,33}$ which lead to an XC vector potential. Up to now these effects have been considered in form of complicated interpolations. 34.35 .36 This problem will be tackled in a forthcoming paper.

\section{ACKNOWLEDGEMENT}

A. W. thanks the the RRZE Erlangen and the DFG (Ro 522/19-1).

1 P. Hohenberg and W. Kohn, Phys. Rev. 136, B 864 (1964).

2 W. Kohn and L. J. Sham, Phys. Rev. 140, A 1133 (1965).

3 U. von Barth and L. Hedin, J. Phys. C 5, 1629 (1972).

4 U. K. Rajagopal and J. Callaway, Phys. Rev. B 7, 1912 (1973). 
5 G. Ortiz, D. M. Ceperley, and R. M. Martin, Phys. Rev. Lett. 71, 2777 (1993).

6 M. Koskinen, M. Manninen, and S. M. Reimann, Phys. Rev. Lett. 79, 1389-1392 (1997).

7 D. G. Austing, S. Sasaki, S. Tarucha, S. M. Reimann, M. Koskinen, and M. Manninen, Phys. Rev. B 60, 11514 (1999).

8 K. Hirose and N.S. Wingreen, Phys. Rev. B 59, 4604 (1999).

9 O. Steffens and M. Suhrke, Phys. Rev. Lett. 82, 3891 (1999).

10 A. Wensauer, O. Steffens, M. Suhrke, and U. Rössler, Phys. Rev. B 62, 2605 (2000).

11 A. Wensauer, J. Kainz, M. Suhrke, and U. Rössler, phys. stat. sol. (b) 224, 675 (2001).

12 M. Ciorga, A. Wensauer, M. Pioro-Ladriere, M. Korkusinski, J. Kyriakidis, A. S. Sachrajda, and P. Hawrylak, Phys. Rev. Lett. 88, 256804 (2002).

13 A. Wensauer, M. Korkusinski, and P. Hawrylak, Phys. Rev. B 67, 035325 (2003).

14 B. Tanatar and D. M. Ceperley, Phys. Rev. B 39, 5005 (1989).

15 C. Attaccalite, S. Moroni, P. Gori-Giorgi, and G. B. Bachelet, Phys. Rev. Lett. 88, 256601 (2002).

16 C.-O. Almbladh and A. C. Pedroza, Phys. Rev. A 29, 2322, (1984).

17 F. Aryasetiawan and M. J. Stott, Phys. Rev. B 38, 2974 (1988).

18 A. Holas and N. H. March, Phys. Rev. A 44, 5521 (1991).

19 A. Görling, Phys. Rev. A 46, 3753 (1992).

20 Y. Wang and R. G. Parr, Phys. Rev. A 47, 1591 (1993).

21 Q. Zhao and R. C. Morrison and R. G. Parr, Phys. Rev. A 50, 2138 (1994).

22 R. van Leeuwen and E. J. Baerends, Phys. Rev. A 49, 2421 (1994).

23 D. J. Tozer, V. E. Ingamells, and N. C. Handy, J. Chem. Phys. 105, 9200 (1996).

24 D. J. Tozer, N. C. Handy, and W. H. Green, Chem. Phys. Lett. 273, 183 (1997).

25 A. K. Rajagopal and J. C. Kimball, Phys. Rev. B 15, 2819 (1977).

26 J. P. Perdew and Y. Wang, Phys. Rev. B 45, 13244 (1992).

27 M. Seidl, University of Regensburg, unpublished (2001).

28 S. M. Reimann, M. Koskinen, and M. Manninen, Phys. Rev. B 62, 8108 (2000).

29 A. Wensauer, M. Korkusinski, and P. Hawrylak, submitted to Phys. Rev. B, 2003.

30 O. Steffens, U. Rössler, and M. Suhrke, Europhys. Lett. 42, 529 (1998).

31 K. Capelle and G. Vignale, Phys. Rev. Lett. 86, 5546 (2001).

32 G. Vignale and M. Rasolt, Phys. Rev. Lett. 59, 2360 (1987). 
33 G. Vignale and M. Rasolt, Phys. Rev. B 37, 10685 (1988).

34 D. Levesque, J. J. Weis, and A. H. MacDonald, Phys. Rev. B 30, 1056 (1984).

35 G. Fano and F. Ortolani, Phys. Rev. B 37, 8179 (1988).

36 M. Rasolt and F. Perrot, Phys. Rev. Lett. 69, 2563 (1992).

37 M. Taut, J. Phys. A 27, 1045 (1994).

38 C. Filippi, C. J. Umrigar, and M. Taut, J. Chem. Phys. 100, 1290 (1994). 
FIG. 1: Comparison of $\mathrm{TC}^{14}, \mathrm{AMGB}^{15}$, and $\mathrm{ISI}^{27}$ parameterizations as a function of density parameter and polarization. In (a) and (b) the relative deviation $\varepsilon_{\mathrm{XC}}^{\mathrm{AMGB}} / \varepsilon_{\mathrm{XC}}^{\mathrm{TC}}-1$ between $\mathrm{TC}$ and AMGB is plotted on different scales for $r_{s}$. (c) and (d) show the difference between ISI and TC $\left(\varepsilon_{\mathrm{XC}}^{\mathrm{AMGB}} / \varepsilon_{\mathrm{XC}}^{\mathrm{TC}}-1\right)$ and ISI and $\mathrm{AMGB}\left(\varepsilon_{\mathrm{XC}}^{\mathrm{AMGB}} / \varepsilon_{\mathrm{XC}}^{\mathrm{TC}}-1\right)$.

FIG. 2: Comparison of GS densities from ED and DFT for a fully polarized eight-electron system ( $L=0, S=4)$ with pronounced differences in the density extrema. The three DFT curves with TC, AMGB, and ISI parameterization lie so close that they cannot be resolved.

FIG. 3: Numerical results of an unpolarized six-electron system with $L=S=0$ and confinement potential $3.32 \mathrm{meV}$. In (a) we show the GS density from ED in (a) as a function of the radius. It is reproduced by the XC potential (black, dashed line) in (b) which gives rise to the extracted $\mathrm{XC}$ energy density (black, dashed line) in (c). In (d) the extracted XC energy density is plotted versus the density parameter $r_{s}$ (black, dashed line). The redundant structure is a conseuquence of the density profile (see text). The grey, solid line in (b), (c), and (d) is the corresponding result calculated with TC parameterization. 
FIG. 4: Summary of extracted XC energy densities for unpolarized systems (six electrons, different confinement potentials) as a function of $r_{s}$. We plotted the deviation of the AMGB, the ISI, and the extracted XC energy densities from the TC parameterization. The results from the density maximum and the dot center are upper and lower bounds for the calculated $\varepsilon_{\mathrm{XC}}$-values. For small $r_{s}$ we find a systematic correction towards smaller XC energy densities.

FIG. 5: Summary of extracted XC energy densities for polarized systems (six (a) and eight (b) electrons, different confinement potentials) as a function of $r_{s}$. We plotted the deviation of the AMGB, the ISI, and the extracted XC energy densities from the TC parameterization. The results from the density extrema represent upper and lower bounds for the calculated $\varepsilon_{\mathrm{XC}}$-values. (c) shows the combined results for six and eight electrons and different confinement strength. For both electron numbers, we find consistent and systematic corrections towards smaller XC energy densities at small density parameters.

FIG. 7: Summary of extracted XC energy densities for partially polarized systems (combined results for four and six electrons, different confinement potentials) as a function of $r_{s}$ and polarization $\xi=0.2(\mathrm{a}), 0.4(\mathrm{~b}), 0.6(\mathrm{c})$ and $0.8(\mathrm{~d})$. We plotted the deviation of the AMGB, the ISI, and the extracted XC energy densities from the TC parameterization. For all systems we find consistent and systematic corrections towards smaller XC energy densities at small density parameters.

FIG. 6: Numerical results of a partially polarized four-electron system with $L=0, S=1$ and confinement potential $3 \mathrm{meV}$. In (a) and (b) we show the GS densities from ED for up- and downspins as a function of the radius. They are reproduced by the XC potentials (black, dashed lines) in (c) which give rise to the extracted XC energy density (black, dashed line) in (d). The grey, solid line in (b), (c), and (d) is the corresponding result calculated with TC parameterization. 

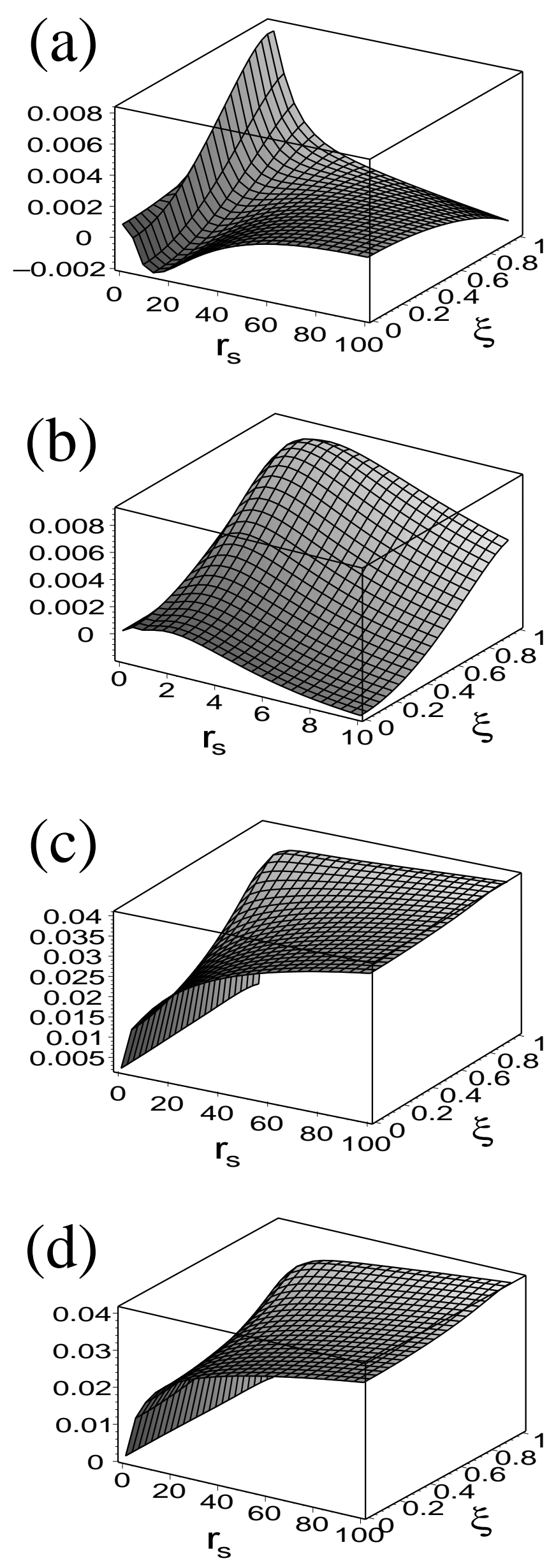

Fig. 1, Wensauer, PRB 


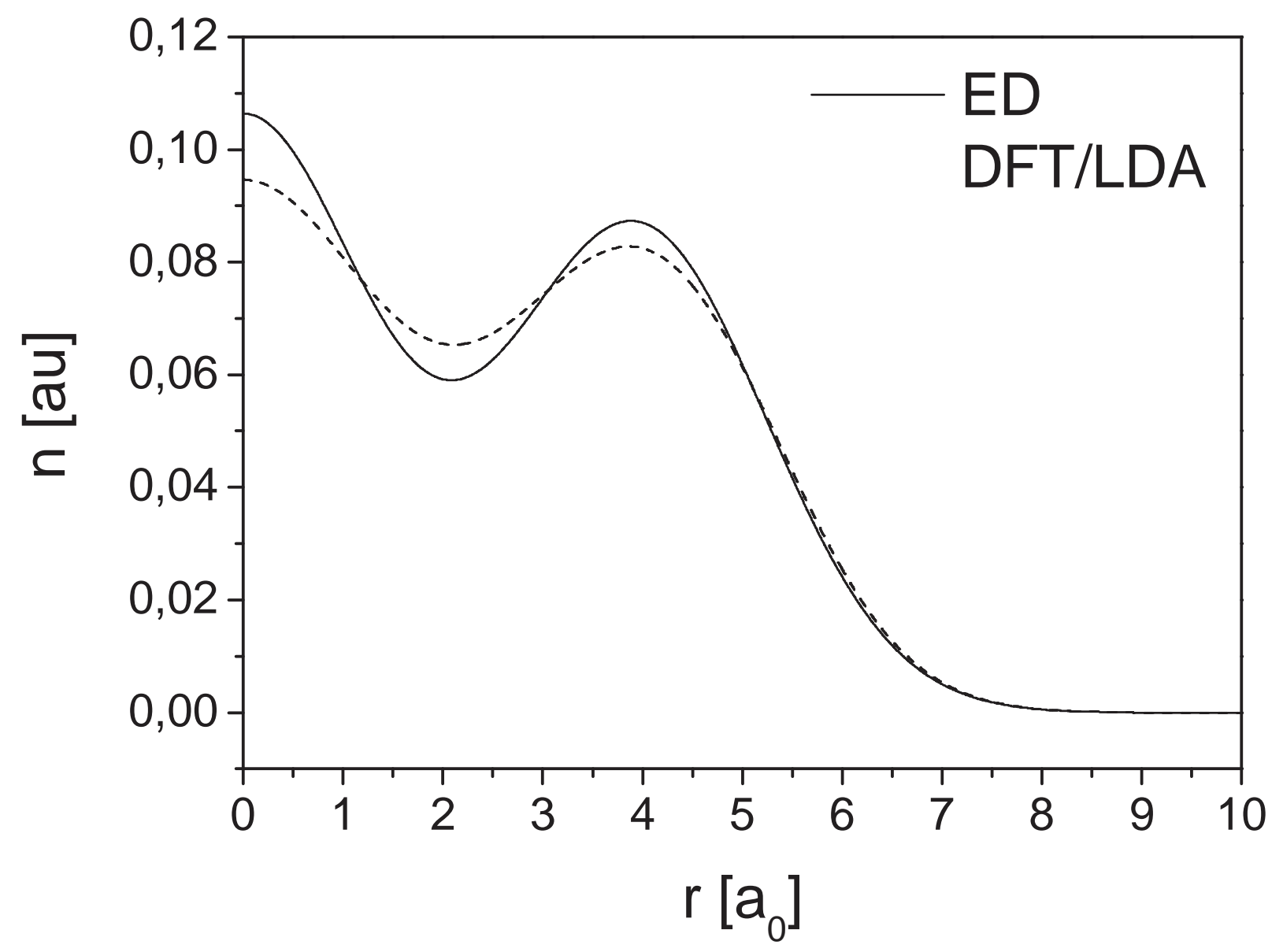

Fig. 2, Wensauer, PRB 

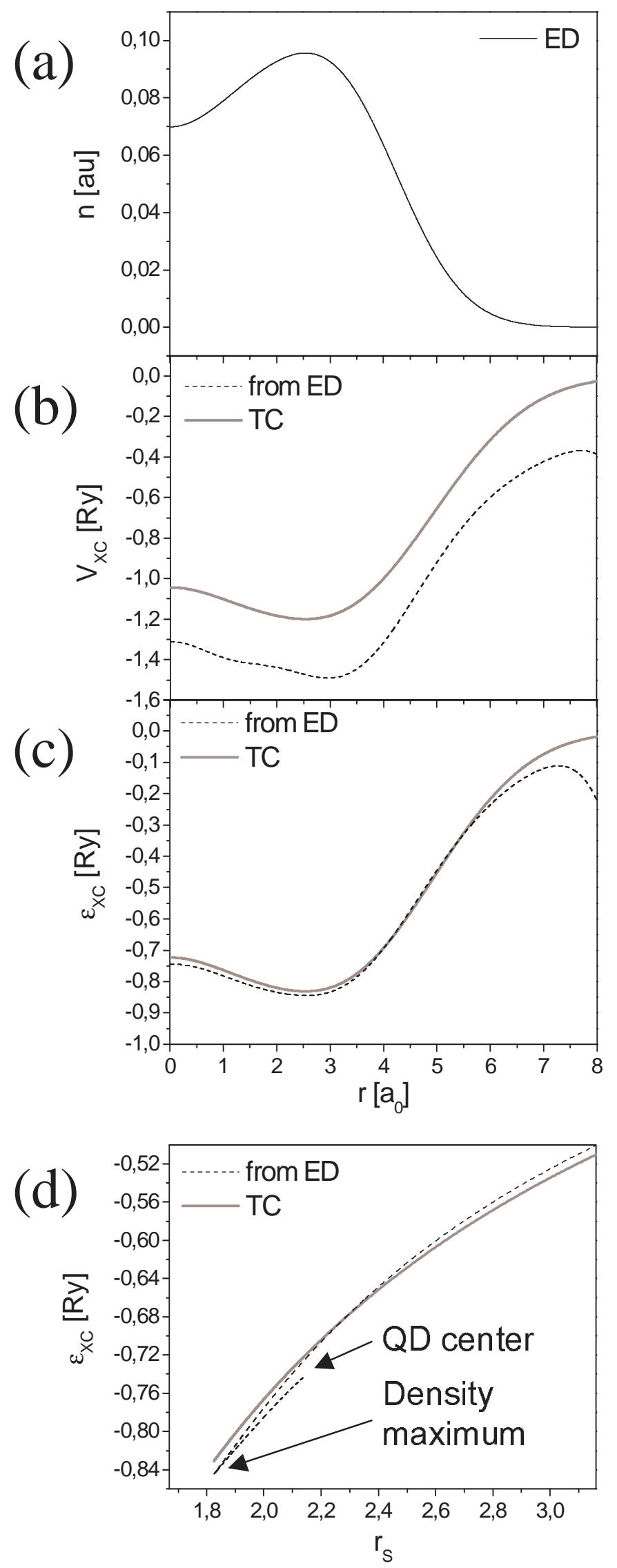

Fig.3, Wensauer, PRB 


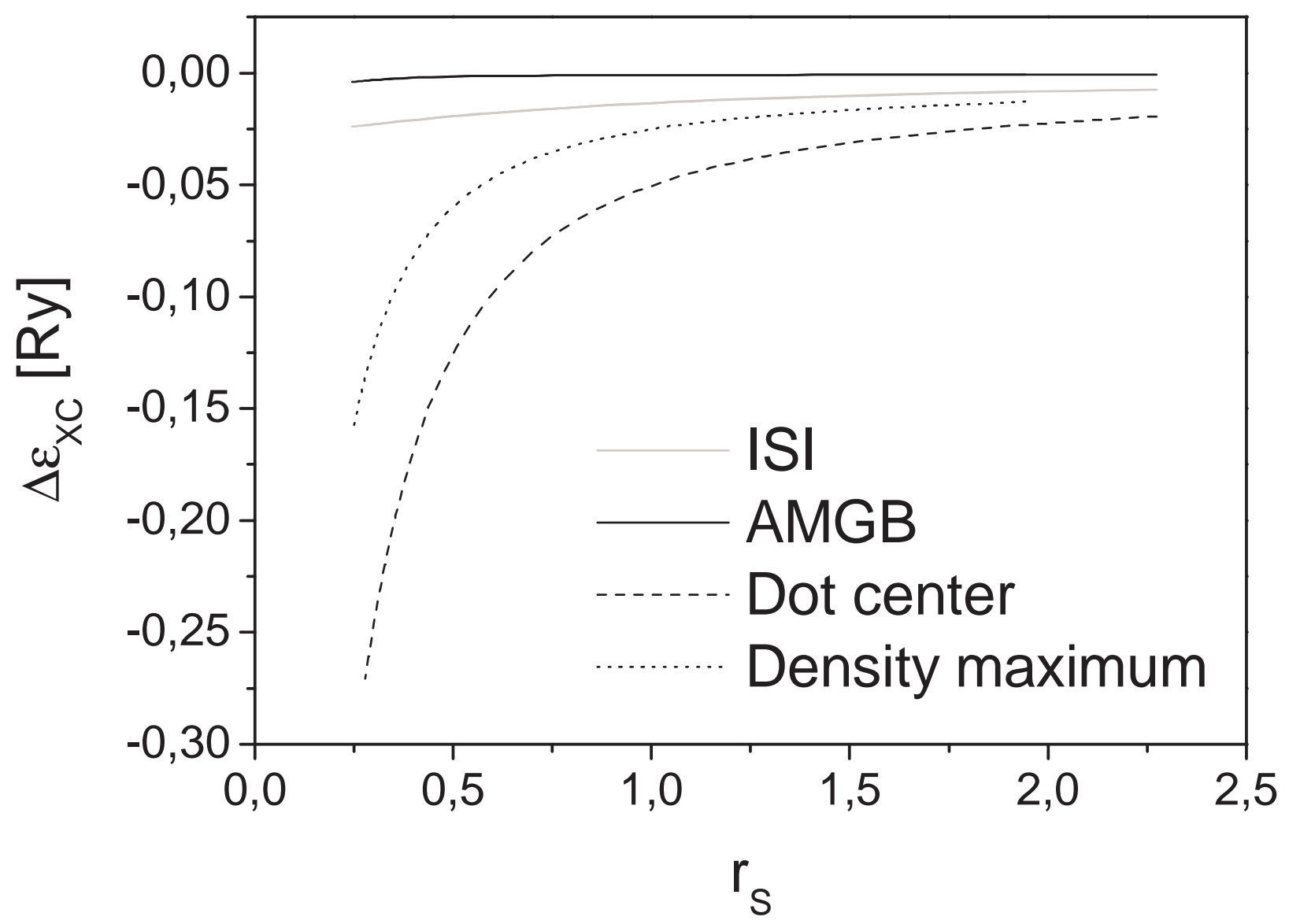

Fig. 4, Wensauer, PRB 


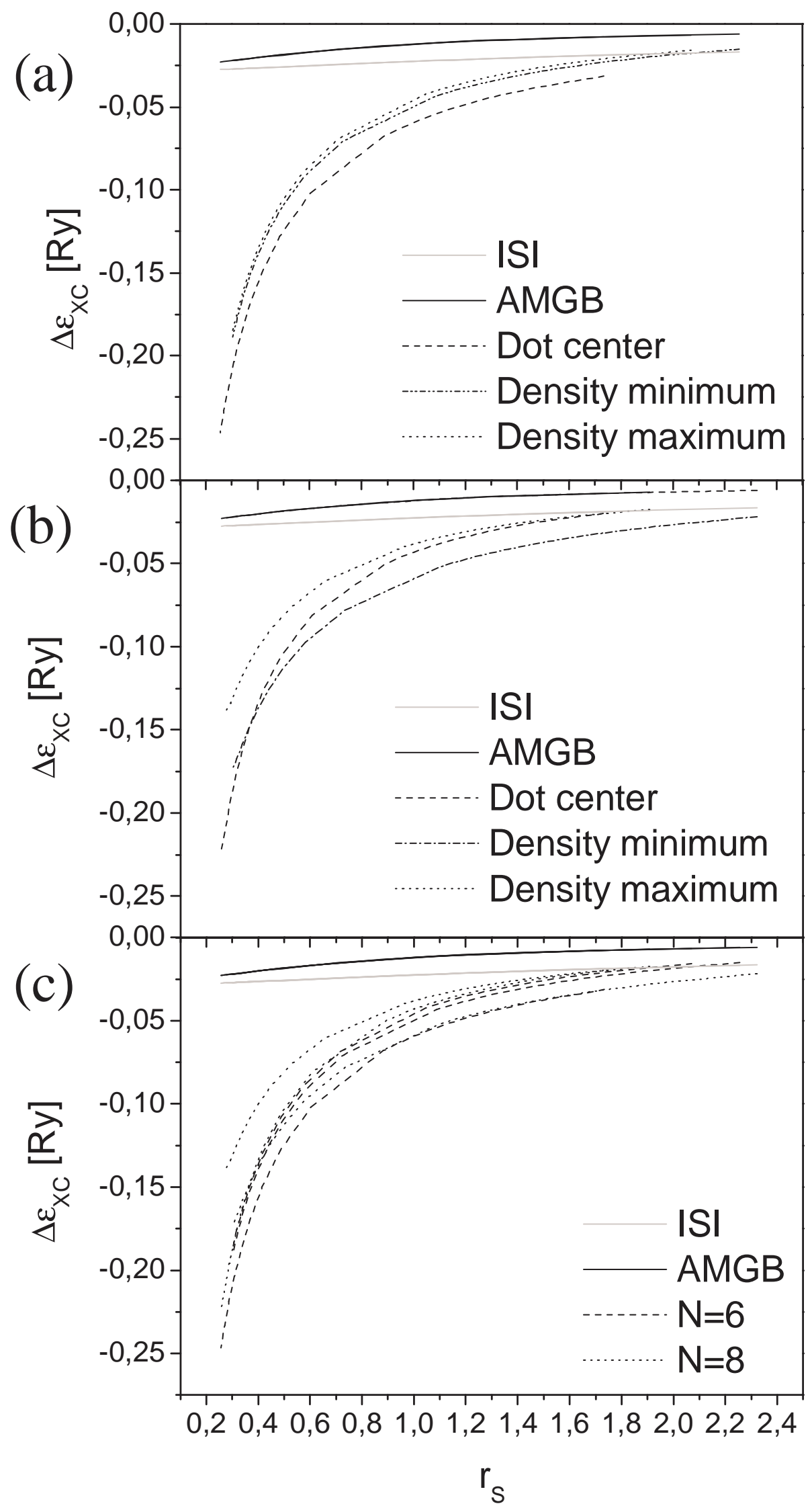

Fig. 5, Wensauer, PRB 


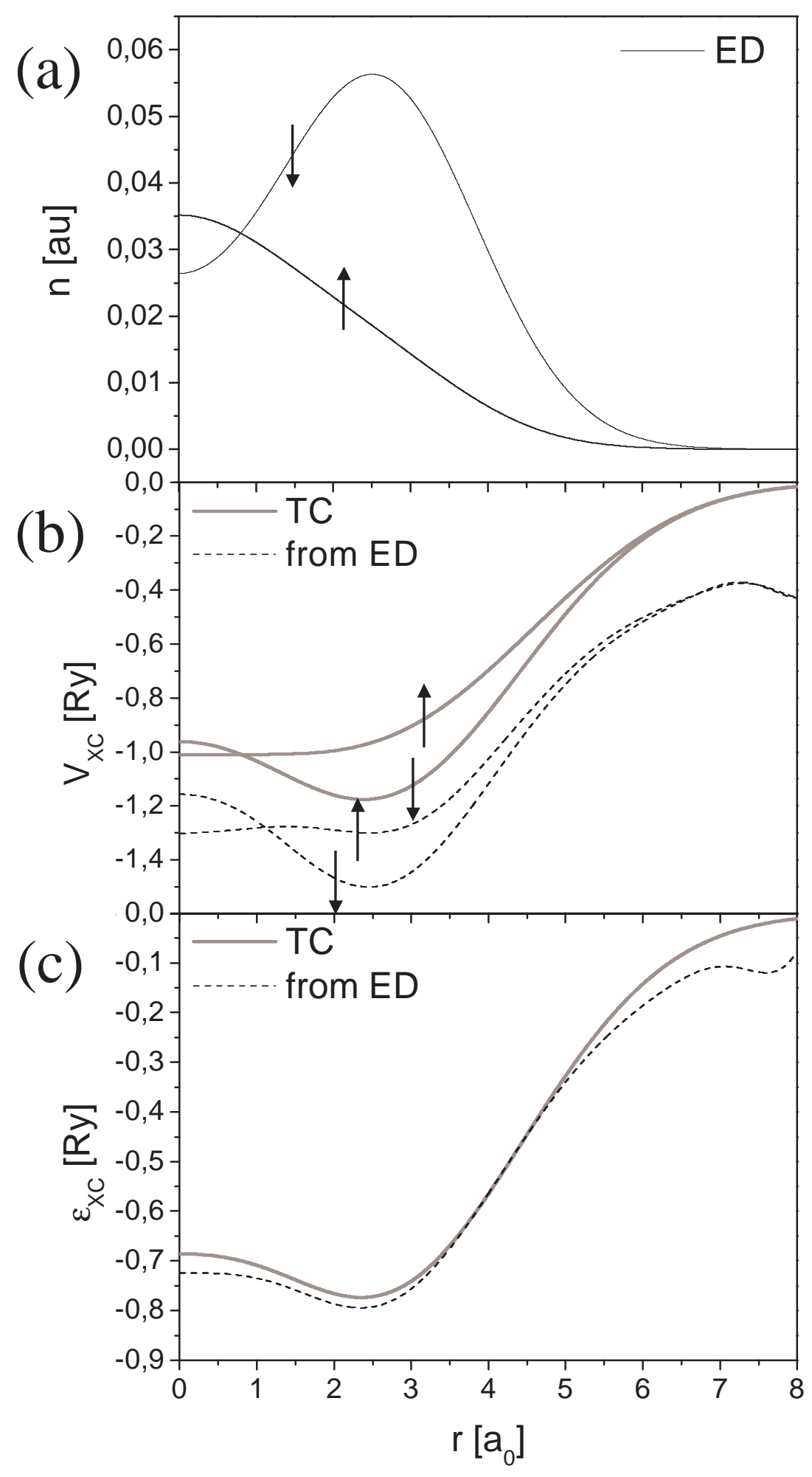

Fig. 6, Wensauer, PRB 


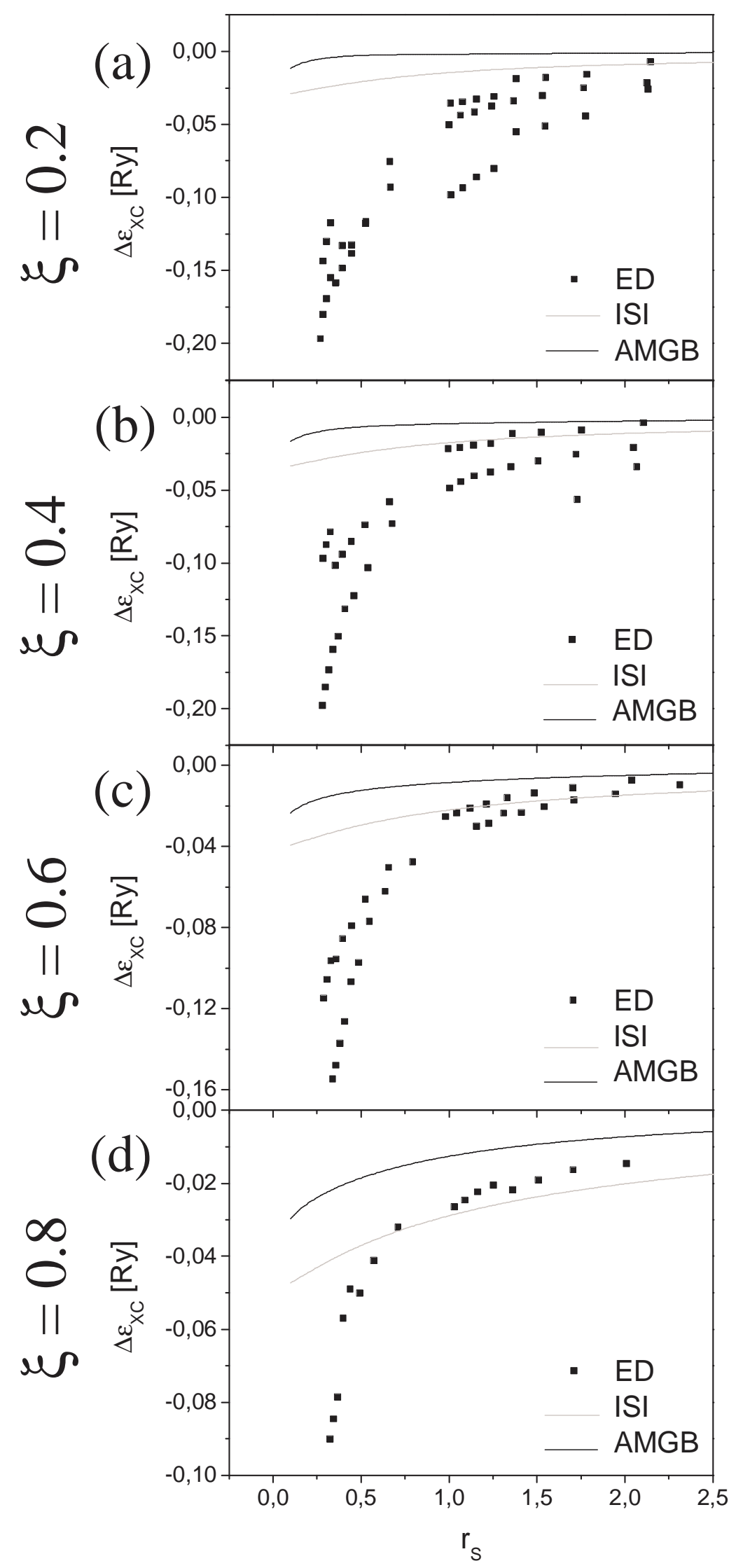

Fig. 7, Wensauer, PRB 